\title{
Realistic Modeling of Water Droplets for Monocular Adherent Raindrop Recognition using Bézier Curves
}

\author{
Martin Roser, Julian Kurz and Andreas Geiger \\ Department of Measurement and Control \\ Karlsruhe Institute of Technology (KIT) \\ D-76131 Karlsruhe, Germany
}

\begin{abstract}
In this paper, we propose a novel raindrop shape model for the detection of view-disturbing, adherent raindrops on inclined surfaces. Whereas stateof-the-art techniques do not consider inclined surfaces because they assume the droplets as sphere sections with equal contact angles, our model incorporates cubic Bézier curves that provide a low dimensional and physically interpretable representation of a raindrop surface. The parameters are empirically deduced from numerous observations of different raindrop sizes and surface inclination angles. It can be easily integrated into a probabilistic framework for raindrop recognition, using geometrical optics to simulate the visual raindrop appearance. In comparison to a sphere section model, the proposed model yields an improved droplet surface accuracy up to three orders of magnitude.
\end{abstract}

\section{Introduction}

Outdoor navigation and surveillance demand for reliable and robust computer vision algorithms. They have to meet stringent conditions concerning disturbances caused by arbitrary weather conditions. In fact, there are various atmospheric influences which restrict the usability of these systems such as fog, rain or snow. Especially in rainy weather it is often the case that adherent waterdrops on the lens-protecting glass disturb the view of a camera. Although a lot of research has been pursued in robotics [16], computer vision $[5,7,11]$ and for driver assistance $[9,12,19]$, raindrop detection still remains a challenging task. This might be for several reasons: Water droplets on a glass surface exhibit a large variety in shape and size. Transparency makes their appearance highly dependent on the image background. Moreover, water droplets on the protecting glass of a camera are subject to severe out-of-focus blur which lowers their distinguishability from the scene background.

Recent work on raindrop detection $[8,12]$ assumes a simple sphere section for modeling the droplet boundary. Especially on tilted planes where gravity causes an unidirectional droplet deformation, this assumption does not hold. While high-order polynomials are more adequate, a physical interpretation of the fitted parameters is hard.

In this paper we propose a novel raindrop shape model, that provides a physically interpretable parameter set of low dimensionality. Our main contribution is a model based on cubic Bézier curves. We provide a broad validation of the model parameters and show, that the shape deviation between fitted model and real droplet will be significantly decreased compared to state-of-the-art sphere section models. The proposed shape model can be easily integrated into existing raindrop recognition frameworks. 


\section{Related Work}

The visual effects of rain are manifold and complex. Water droplets in the atmosphere lead to contrast attenuation in the far-field of the camera, whereas falling raindrops produce sharp intensity changes in image sequences. Adherent raindrops in front of the camera lens disturb the view from the camera and light reflections on the droplet surfaces additionally deteriorate computer vision algorithms.

Related work on dynamic weather effects like the appearance of falling raindrops in image sequences has been performed by $[5,7]$. They studied the influence of falling raindrops on the image acquisition process and introduced a photometric model for spherical raindrops in the atmosphere that is used for enhanced video processing like removing rain from image sequences [4] or rain streak rendering [6].

In the targeted context of outdoor navigation and surveillance, falling raindrops and rain streaks can be considered as atmospheric noise and are not the dominant disturbing effect. Stronger limitations are imposed by adherent water droplets on the glass surface covering the camera lens. Kurihata et al. [9] used a machine learning approach with raindrop templates to detect raindrops on windshields from inside a moving vehicle. Results within the sky area were promising, whereas the proposed method produced a large number of false positives within the non-sky regions of the image where raindrop appearance modeling becomes more challenging. In this work, in contrast, we aim to accurately exploit the physical relationship between droplets shape and their appearance. Zhang et al. [19] combined a wavelet transform for image blur detection with motion analysis using cumulative differences to recognize optical contaminations close to the camera. Their approach works well for rigid, opaque contaminations but fails in the presence of raindrops because their appearance strongly depends on the scene background. Yamashita et al. exploited hardware constraints like multiple cameras $[13,18]$ or pan-tilt surveillance cameras $[16,17]$ with known yaw rates in order to bypass the challenge of modeling the complex optical behavior of raindrops. Roser et al. $[8,12]$ simulated spherical droplets on a glass surface using geometrical optics and out-of-focus blur for the task of raindrop detection. However, they lack a realistic shape parametrization of droplets that in practice are subject to gravity.

The remainder of this paper is structured as follows: Section 3 discusses the propagation of light rays through a droplet and shows how it can be used for raindrop detection. In Section 4 we proposes a raindrop shape model based on a Bézier curve representation. Validation results on real data and a comparison to a sphere section model [8] are given in Section 5.

\section{Raindrop Recognition}

Given a set of $n$ artificial raindrop hypotheses $\mathbf{D}_{\mathbf{1}}, \ldots, \mathbf{D}_{\mathbf{n}}$ for different image positions and presumed drop radii, the raindrop recognition task can be formulated as computing the MAP estimate of the conditional probability

$$
p(\mathbf{d} \mid \mathbf{z}) \propto p(\mathbf{d}) p(\mathbf{z} \mid \mathbf{d})
$$

with respect to the pattern $\mathbf{d} \in\left\{\oslash, \mathbf{D}_{\mathbf{1}}, \ldots, \mathbf{D}_{\mathbf{n}}\right\}$. Here $\oslash$ is a background pattern that models the case where the image region is not disturbed by a raindrop and $\mathbf{z}(u, v, r)$ 


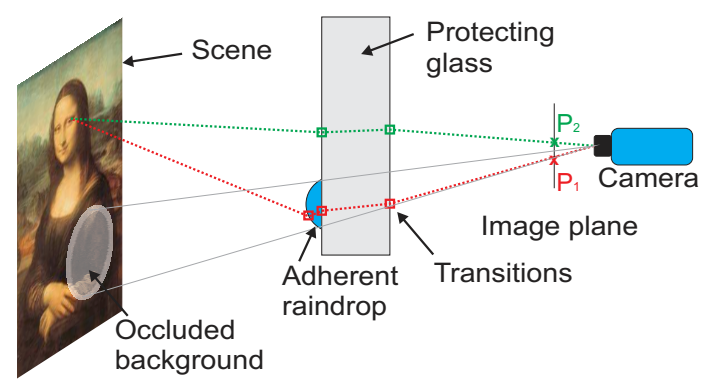

(a) $3 \mathrm{D}$ raindrop refraction model

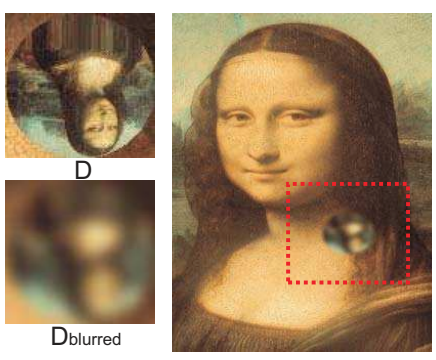

(b) Artificial raindrop pattern

Fig. 1. Droplet refraction model. (a) depicts the image formation process in the presence of raindrops on a protecting glass in front of the camera, using geometrical optics. In (b) an artificial raindrop pattern $\mathbf{D}$ is rendered by tracing the light rays through the raindrop to the background and composing all found background pixels. For demonstration purposes, an additional out-offocus blur is applied and the blurred pattern $\mathbf{D}_{\text {blurred }}$ is added to the original image.

denotes the observation at position $u, v$ with radius $r$ in form of local image statistics. It can be achieved densely as wells as from preselected points of interest, like CenSurE [1] or SURF [2]. Fairly standard cost measures such as the Sum-of-Absolute Differences (SAD) or the Sum-of-Squared Differences (SSD) are applied for modeling the observation likelihood $p(\mathbf{z} \mid \mathbf{d})$. The prior may model the occurrence probability for different raindrop sizes in various adverse weather conditions in an empirical Bayesian perspective according to [15].

Raindrop hypotheses for any circular region $\mathbf{x}=(u, v, r)^{T}$ are achieved from observed points in the environment, using geometrical optics. As depicted in Fig. 1(a), a light ray emanating from a point in the environment will be refracted by the raindrop and the protecting glass surface multiple times and reaches the camera sensor at point $\mathbf{P}_{1}$. Unless the raindrop does not occlude this environment point, it will be sensed a second time at point $\mathbf{P}_{2}$. Note, that the droplet acts as a convex lens with a small focal length. Hence, for typical application in navigation and surveillance it is ensured that only a minor part of the environment points are occluded by the raindrop (see Fig. 1(a)). An accurate geometric relationship between $\mathbf{P}_{\mathbf{1}}$ and $\mathbf{P}_{\mathbf{2}}$ can be derived using Snell's law of refraction as shown in Fig. 1(b). Note, that the refraction on the protecting glass occurs with respect to the (constant) plane normal $n_{p}$ of the protecting glass, whereas a general drop surface $\mathbf{S}$ exhibits a normal field $\mathbf{N}_{\mathbf{S}}$ that can be deduced from the chosen drop parametrization. Whereas $[8,12]$ use simple sphere sections that are in general 3D surfaces of constant curvature as depicted in Fig. 2(a), this model approximates the raindrop shape only insufficiently and results in a high model deviation especially when dealing with tilted glass surfaces. For this reason, we derive a raindrop shape model regarding numerous tilt angles and drop sizes by using two orthogonal oriented Bézier curves as illustrated in Fig. 2(b). 


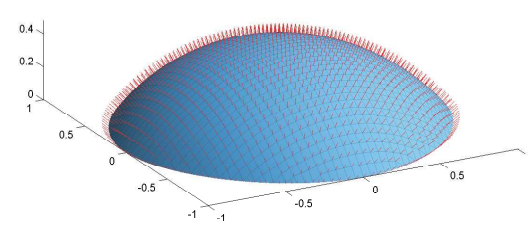

(a) Sphere section model

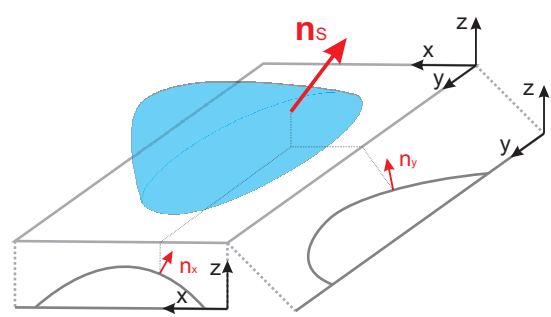

(b) Proposed shape model

Fig. 2. Raindrop surface models. (a) shows the droplet surface and its surface normals for a sphere section model. In (b) a 3D model is created by superposing two orthogonal Bézier curves.

\section{Raindrop Shape Model}

\subsection{Bézier Representation}

Droplets on a horizontally aligned surface are symmetrical and have equal contact angles. When neglecting any gravity, they can be characterized adequately, using the sphere section model as described in $[8,12]$. However, gravity leads to a flattened raindrop surface shape which results in an inaccurate droplet modelling when assuming sphere sections. On tilted surfaces the sphere section model assumption is violated even more, because the unsymmetrically applied gravity force will shift the droplet centroid towards the declining direction, which yields different contact angles and a distinctly bellied shape as illustrated in Fig. 5.

The shape of a raindrop can be described by parametric functions, like polynomials of arbitrary order, Taylor polynomials or Bézier curves [3]. Here we employ Bézier curves, since they describe real water droplets accurately and they provide an intuitive, low-dimensional parameter set with a credible physical interpretation. This makes the verification of the model and an approximation for different angles and drop volumes more transparent than interpreting the coefficients of a polynomial fit.

A Bézier curve of the degree $n$ is characterized by a control polygon consisting of $n+1$ Bézier points $\left(\mathbf{P}_{i}\right)_{i=0}^{n}, \mathbf{P} \in \mathbb{R}^{2}$. It is defined in an interval $t \in[0 \ldots 1]$ as

$$
\mathbf{C}(t)=\sum_{i=0}^{n} \mathbf{B}_{i, n}(t) \mathbf{P}_{i}
$$

whereas

$$
\mathbf{B}_{i, n}(t)=\left(\begin{array}{c}
n \\
i
\end{array}\right) t^{i}(1-t)^{n-i}
$$

indicates the Bernstein polynomial $i$ of degree $n$ [3].

A cubic Bézier curve $(n=3)$ has sufficient degrees of freedom to describe the raindrop shape well. As depicted in Fig. 3, a capable interpretation of the Bézier points $\left(\mathbf{P}_{i}\right)_{i=0}^{3}$ can be achieved by transforming them to the contact angles $\alpha_{1}, \alpha_{2}$ of the droplet that are originated from physics of boundaries and the weight factors $w_{1}, w_{2}$ 


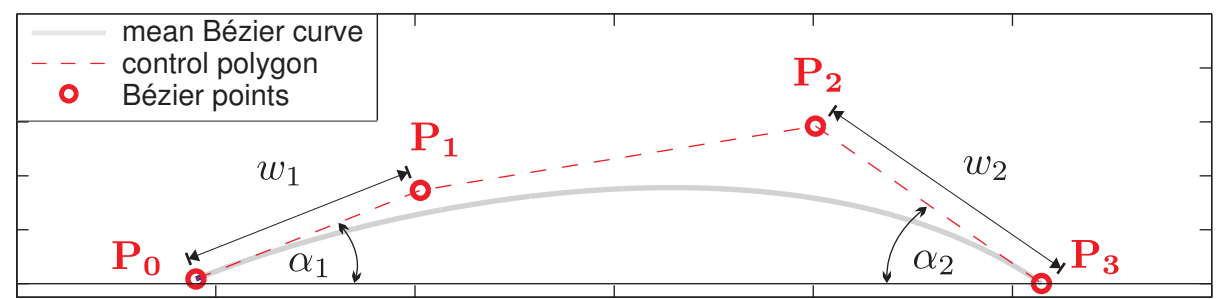

Fig. 3. Cubic Bézier curve representation. The Bézier points are transformed physically interpretable: $\alpha_{1}, \alpha_{2}$ represent the droplets contact angles and the weight factors $w_{1}, w_{2}$ are related to the influence of gravity for inclined surfaces.

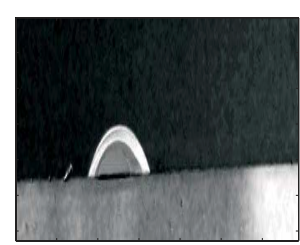

(a)

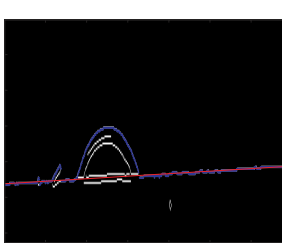

(b)

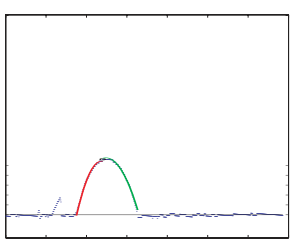

(c)

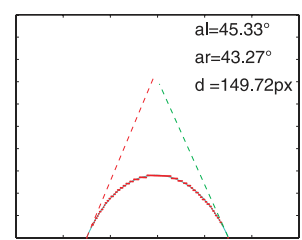

(d)

Fig. 4. Image processing for drop shape extraction. (a) shows the original image taken in the experimental setup. A distinction between surface plane and raindrop points is performed by RANSAC line fitting in the Canny image (b). In order to remove further outliers, two second order polynomials are fitted robustly to the left (red) and right (green) side of the raindrop (c). Finally, least squares Bézier curve fitting is performed on all inlier points (d).

that are related to the centroid shift due to gravity.

$$
\begin{aligned}
& \left.\alpha_{1}=\angle \overline{\mathbf{P}_{\mathbf{0}} \mathbf{P}_{\mathbf{1}}}, \overline{\mathbf{P}_{\mathbf{0}} \mathbf{P}_{\mathbf{3}}}\right) \\
& \left.\alpha_{2}=\angle \overline{\mathbf{P}_{\mathbf{2}} \mathbf{P}_{\mathbf{3}}}, \overline{\mathbf{P}_{0} \mathbf{P}_{\mathbf{3}}}\right) \\
& w_{1}=\overline{\mathbf{P}_{\mathbf{0}} \mathbf{P}_{\mathbf{1}}} \\
& w_{2}=\overline{\mathbf{P}_{\mathbf{2}} \mathbf{P}_{\mathbf{3}}}
\end{aligned}
$$

Finally, the curvature normals of two orthogonal, cubic Bézier curves form a 3D droplet surface $\mathbf{S}$ as illustrated in Fig. 2(b)

$$
\mathbf{n}_{\mathbf{S}}=\frac{1}{\left\|\left(n_{x}, 0,1\right)^{T}+\left(0, n_{y}, 1\right)^{T}\right\|}\left(\left(\begin{array}{c}
n_{x} \\
0 \\
1
\end{array}\right)+\left(\begin{array}{c}
0 \\
n_{y} \\
1
\end{array}\right)\right),
$$

where one curvature represents the side view with the inclination angle of the lensprotecting glass and the other representing the top view with $\theta=0^{\circ}$.

\subsection{Bézier curve fitting}

In order to characterize and describe the drop shape in terms of cubic Bézier curves, we performed an image pre-processing as described briefly in the following paragraph. 
An overview of the image processing and curve fitting methods can be found in Fig. 4. The first step of extracting the drop shape is to take raw observations from the canny edge image (Fig. 4(b)). A robust RANSAC line fitting approach estimates the remaining glass surface direction and hence compensates small errors due to inaccuracies in the angular arrangement of glass plate and camera. In order to further remove outliers from the measurements, two parabolas were fitted through the remaining points, using RANSAC: one from the maximum to the left side (red line in Fig. 4(c)) and one to the right side (green line in Fig. 4(c)). Note, that we do not use the parameters of the parabola fits directly because the shape is neither described consistently nor interpretable in a physical way. Instead, a combination of all inliers gives a set of points that is used for the subsequent Bézier curve fitting as shown in Fig. 4(d). The Bézier curve fitting is performed in a least squares sense [14] by splitting (2) into two independent equations for the $x$ and $y$ coordinates

$$
\begin{aligned}
& x=a_{x} t^{3}+b_{x} t^{2}+c_{x} t+d_{x} \\
& y=a_{y} t^{3}+b_{y} t^{2}+c_{y} t+d_{y},
\end{aligned}
$$

and computing the Bézier points $\left(\mathbf{P}_{i}\right)_{i=0}^{3}$ by comparing coefficients to (2). The factor $t \in[0 \ldots 1]$ corresponds to the normalized curvature length. For a curve described by $N$ points $t$ is approximated by

$$
t(n)=\frac{\sum_{k=1}^{n} \sqrt{\Delta x(k)^{2}+\Delta y(k)^{2}}}{\sum_{l=1}^{N} \sqrt{\Delta x(l)^{2}+\Delta y(l)^{2}}},
$$

where $\Delta x$ and $\Delta y$ are the differences between two neighboring points.

Repeating the experiments $M$ times for each drop volume and inclination angle configuration, we receive $M$ different Bézier curve parameterizations. A mean Bézier curve is finally achieved by computing the mean of each Bézier point $\left(\mathbf{P}_{i}\right)_{i=0}^{3}$ :

$$
\mathbf{P}_{\mathbf{i}}=\sum_{k=1}^{M} \frac{\mathbf{P}_{\mathbf{i}}{ }^{k}}{M} .
$$

\section{Results}

For all experiments, a digital camera was mounted next to a tiltable glass plate to capture the shape of water droplets of different sizes under multiple inclination angles. We used an Eppendorf Research Plus pipette for all experiments in order to guarantee a precise but adjustable drop volume size. In the experimental setup all drops are illuminated by a lamp in front of a dark background to achieve a good contrast and ensure reliable shape extraction. As input for finding an empirical description of water droplets on a flat surface, multiple images with different drop sizes and surface inclination angles were taken. The chosen drop volumes for our test series were 5, 10, 15 and $20 \mu l$ and the inclination angles of the glass plate were $0^{\circ}, 25^{\circ}, 30^{\circ}, 35^{\circ}$ and $40^{\circ}$. The chosen 


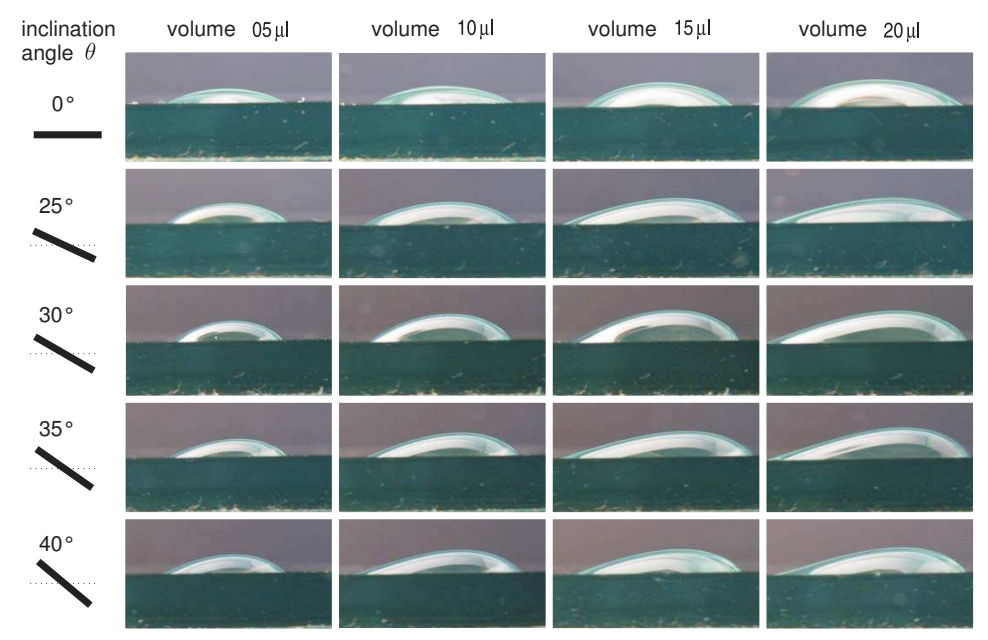

Fig. 5. Experiments. Sample imagery for manifold drop volumes and surface inclination angles. For model estimation the mean fit of 20 images for each drop volume and inclination angle setting is used.

drop volumes in $\mu l$ correspond to $1.06,1.34,1.53$ and $1.7 \mathrm{~mm}$ drop radii of falling raindrops, which was motivated by [7] who proposes probable raindrop radii between $0.5-2.5 \mathrm{~mm}$. The experiments were repeated 20 times for each drop volume and inclination angle configuration. Hence, 400 raindrop shape images were acquired in total. An overview of the different setup properties and their effects on the droplet shape is depicted in Fig. 5.

The results section is divided into two parts: First, we discuss the estimated raindrop parameters. Then we present a comparison of the proposed model with the sphere section model of [8].

\subsection{Model parameters}

A model capable of generating realistic droplet surfaces demands for a low dimensional parametrization to avoid overfitting. In this section, we discuss the obtained dependencies of the Bézier curve based model with respect to the design parameters (drop volume and inclination angle).

Assuming the raindrop diameter $d=\left|\overline{\mathbf{P}_{0} \mathbf{P}_{3}}\right|$, the upper row in Fig. 6 shows the expected behavior that the drop radius increases with its volume. A tendency of increasing drop diameters for larger inclination angles exists, although it may not be the predominant effect. This phenomenon can be explained from the droplet area that looses its circular shape and develops a predominant direction with increasing inclination angles. Hence, even if the drop volume is not a-priori known like it is the case in image-based raindrop detection tasks, for a given surface inclination angle the volume can be estimated from the observed drop diameter. In principle, the scale of standard multi-scale interest point detectors like SURF [2] would provide sufficient information for that task. 


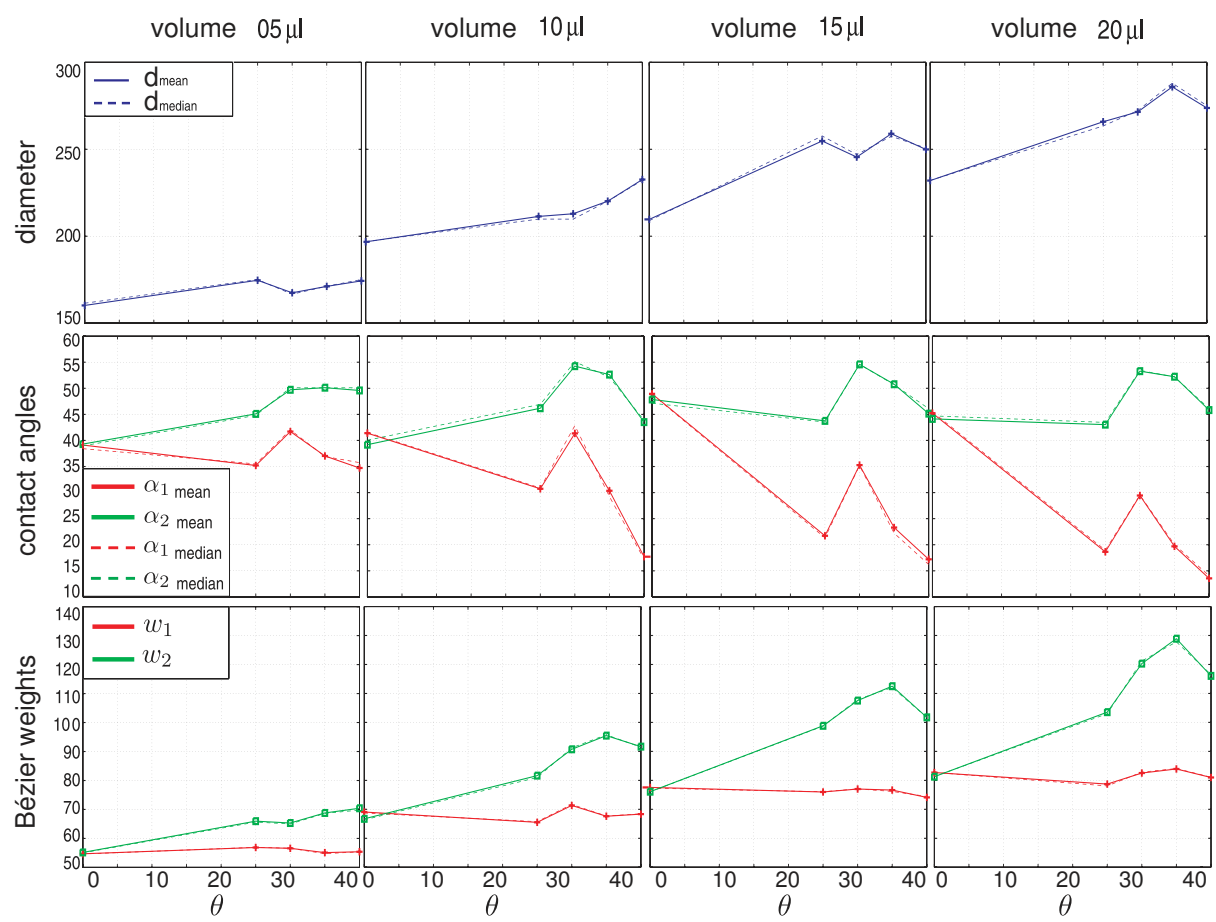

Fig. 6. Model parameter. The first row shows the averaged droplet diameter $d$ as a function of the inclination angle $\theta$ for different raindrop volumes (columns). The second and the third row depict the mean contact angles $\alpha_{1}, \alpha_{2}$ and the mean Bézier weights $w_{1}, w_{2}$, respectively.

Tilting the glass surface leads to a deformation of the drop due to changed gravity influences. For this reason, we expect an increasing difference $\Delta=\left|\alpha_{2}-\alpha_{1}\right|$ between both contact angles. The middle row in Fig. 6 shows the expected behavior, although not all contact angles could be extracted accurately, throughout the experiments. However, $\alpha_{1}$ tends to decrease with increasing inclination angle. $\alpha_{2}$ shows a slight ascent but decreases for $\theta=40^{\circ}$. This can be explained by having a deeper look at the performed experiments. We are only interested in stationary droplets. For $\theta \approx 40^{\circ}$, the drop begins rinsing down and hence we could not acquire representative imagery data.

The Bézier weight $w_{1}$ remains constant for varying inclination angle and ascends with increasing drop volume. For the right side $w_{2}$ increases with inclination angle and drop volume while for angles $\theta \approx 40^{\circ}$ the drop begins to move, again. As discussed above, for these inclination angles, no reliable conclusion can be drawn.

In conclusion, a physically correct droplet shape can be derived, as soon as the inclination angle and the drop volume are given. The drop volume can be deduced from the observed raindrop diameter, whereas the surface inclination angle is given by the defined camera mounting. This makes the proposed droplet shape model applicable for an image-based raindrop detection approach. 


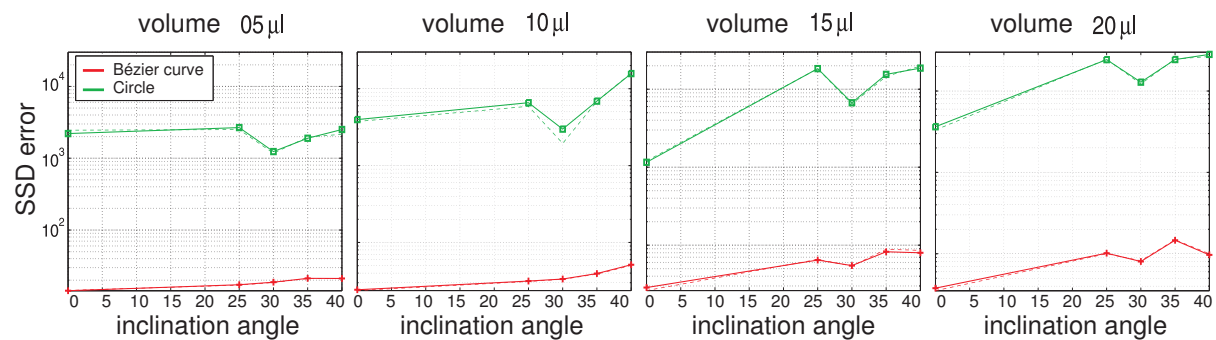

Fig. 7. Model accuracy. (a)-(d) show the SSD error of a sphere section model and the proposed model, using cubic Bézier curves.

\subsection{Comparison}

For comparing the accuracy of the proposed method to state-of-the art, a 2D cut of a sphere section was fitted to the extracted raindrop surface measurements using nonlinear Levenberg-Marquardt optimization [10]. The error measure is defined in terms of Sum-of-Squared Differences (SSD).

Fig. 7 shows the error generated using the sphere section model in comparison to the new Bézier curve based model. Even for flat surfaces $\left(\theta \approx 0^{\circ}\right)$ and small drop volumes, the proposed model has an SSD error which is three order of magnitude smaller. This illustrates the importance to take into account the gravity force which flattens the drop surface. An increasing drop volume and inclination angle lead to unsymmetrical droplet deformation, which emphasizes the advantage of the proposed shape model with respect to the sphere section model.

\section{Conclusion}

In this paper we proposed a novel raindrop shape model based on cubic Bézier curves and showed its potential for its integration in image-based raindrop detection approaches. The model was deduced from numerous experiments on water drops of various volumes on a flat surface with different inclination angles. A physically correct droplet shape could be computed if just the inclination angle and the drop volume were given. The drop volume was deduced from the observed raindrop diameter. This makes the proposed droplet shape model applicable for an image-based raindrop detection approach. Finally, we showed that the shape deviation between the estimated Bézier curve based model and the real droplet was significantly decreased compared to state-of-theart sphere section models.

\section{Acknowledgment}

The authors would like to thank the Karlsruhe School of Optics and Photonics (KSOP). 


\section{References}

1. M. Agrawal, K. Konolige, and M.R. Blas. CenSurE: Center Surround Extremas for Realtime Feature Detection and Matching., volume 5305 of Lecture Notes in Computer Science, pages 102-115. Springer, 2008.

2. H. Bay, T. Tuytelaars, and L. Van Gool. Surf: Speeded up robust features. In European Conference on Computer Vision (ECCV'06), Graz Austria, May 2006.

3. G. Farin. Curves and surfaces for CAGD: a practical guide. Morgan Kaufmann Publishers Inc., San Francisco, CA, USA, 2002.

4. K. Garg and S.K. Nayar. Detection and removal of rain from videos. In IEEE Conference on Computer Vision and Pattern Recognition (CVPR '04), volume I, pages 528-535, Jun 2004.

5. K. Garg and S.K. Nayar. When does a camera see rain? In IEEE International Conference on Computer Vision (ICCV '05), volume 2, pages 1067-1074, Oct 2005.

6. K. Garg and S.K. Nayar. Photorealistic rendering of rain streaks. ACM Transactions on Graphics, Jul 2006.

7. K. Garg and S.K. Nayar. Vision and rain. Internatl. Journal of Computer Vision, 75(1):3-27, 2007.

8. J. Halimeh and M. Roser. Raindrop detection on car windshields using geometricphotometric environment construction and intensity-based correlation. In IEEE Intelligent Vehicle Symposium (IV '09), Xi' an, China, 2009.

9. H. Kurihata, T. Takahashi, I. Ide, Y. Mekade, H. Muraseand Y. Tamatsu, and T. Miyahara. Rainy weather recognition from in-vehicle camera images for driver assistance. In IEEE Intelligent Vehicles Symposium (IV '05), pages 205-210, 2005.

10. D. Marquardt. An algorithm for least-squares estimation of nonlinear parameters. SIAM Journal of Applied Mathematics, 11:431-441, 1963.

11. S.G. Narasimhan and S.K. Nayar. Vision and the atmosphere. International Journal of Computer Vision, 48(3):233-254, 2002.

12. M. Roser and A. Geiger. Video-based raindrop detection for improved image registration. In IEEE Workshop on Video-Oriented Object and Event Classification (in conjunction with ICCV'09), 2009.

13. Y. Tanaka, A. Yamashita, T. Kaneko, and K.T. Miura. Removal of adherent waterdrops from images acquired with a stereo camera system. IEICE - Transactions on Information Systems, E89-D(7):2021-2027, 2006.

14. P. J. G. Teunissen. Adjustment theory: an introduction. Delft University Press, Postbus 98, 2600 MG Delft, 2000.

15. Nuno Vasconcelos and Andrew Lippman. Empirical bayesian em-based motion segmentation. In Proc. of the IEEE Conf. on Computer Vision and Pattern Recognition, Puerto Rico, pages 527-532, 1997.

16. A. Yamashita, I. Fukuchi, T. Kaneko, and K.T. Miura. Removal of adherent noises from image sequences by spatio-temporal image processing. IEEE International Conference on Robotics and Automation (ICRA '08), pages 2386-2391, May 2008.

17. A. Yamashita, T. Harada, T. Kaneko, and K.T. Miura. Removal of adherent noises from images of dynamic scenes by using a pan-tilt camera. IEEE/RSJ International Conference on Intelligent Robots and Systems (IROS '04), 1:437-442 vol.1, Sept.-2 Oct. 2004.

18. A. Yamashita, M. Kuramoto, T. Kaneko, and K.T. Miura. A virtual wiper - restoration of deteriorated images by using multiple cameras. IEEE/RSJ International Conference on Intelligent Robots and Systems (IROS '03), 4:3126-3131 vol.3, Oct. 2003.

19. Y. Zhang, J. Yang, K. Liu, and X. Zhang. Self-detection of optical contamination or occlusion in vehicle vision systems. Journal of Optical Engineering, 47(6):067006, 2008. 